\title{
Mediterranean diet in type 2 diabetes. Reply to Basterra-Gortari FJ, Martínez-González MA [letter]
}

\author{
A. Fraser • D. A. Lawlor • D. Fraser
}

Received: 16 July 2008 / Accepted: 17 July 2008 / Published online: 15 August 2008

(C) Springer-Verlag 2008

Keywords Alanine aminotransferase - Mediterranean diet . Non-alcoholic fatty liver disease $\cdot$ Randomised controlled trial · Type 2 diabetes

\section{Abbreviations \\ MMD modified Mediterranean diet}

To the Editor: We thank Drs Basterra-Gortari and MartínezGonzález for their insightful comments [1] on our recent finding that a modified Mediterranean diet was associated with the greatest benefit in terms of reducing alanine aminotransferase levels among obese patients with diabetes [2]. As they point out, we provided information regarding participants' dietary intake by nutrients and not by food groups. Unfortunately, data of consumption by food groups is not currently available for analysis. However, participants randomised to the modified Mediterranean diet were advised to replace butter with extra virgin olive oil and

\footnotetext{
A. Fraser $(\bowtie) \cdot$ D. A. Lawlor

MRC Centre for Causal Analysis in Translational Epidemiology, Department of Social Medicine, University of Bristol, Oakfield House, 15-23 Oakfield Grove,

Bristol BS8 2BN, UK

e-mail: Abigail.fraser@bristol.ac.uk

\section{Fraser}

Epidemiology Department and S. Daniel Abraham International Center for Health and Nutrition,

Ben Gurion University of the Negev,

Beer-Sheva, Israel
}

olive-based margarine and to include fish, walnuts, peanuts, almonds and avocado in their diet. All three diets included eggs, chicken and turkey breast. Thus, participants were indeed advised to adopt a 'modified functional definition' of the traditional Mediterranean diet; hence, the diet was named a modified Mediterranean diet (MMD).

According to the information collected at 6 months using food frequency questionnaires, participants assigned to the MMD consumed a diet richer in monosaturated fats than did participants assigned to the 2003 American Diabetes Association diet or the low glycaemic index diet. The difference was more modest than that originally aimed for, yet the MMD was still associated with the greatest benefit in terms of a reduction in alanine aminotransferase levels. This finding suggests that, in terms of cardiovascular risk, the type of dietary fat (saturated fat, monounsaturated fat and polyunsaturated fat) may be more important than the quantity of fat consumed.

Duality of interest The authors declare that there is no duality of interest associated with this manuscript.

\section{References}

1. Basterra-Gortari FJ, Martínez-González MA. Mediterranean diet in type 2 diabetes. Diabetologia doi:10.1007/s00125-008-1121-x

2. Fraser A, Abel R, Lawlor DA, Fraser D, Elhayany A (2008) A modified Mediterranean diet is associated with the greatest reduction in alanine aminotransferase levels in obese type 2 diabetes patients: results of a quasi-randomised controlled trial. Diabetologia doi:10.1007/s00125-008-1049-1 